\title{
New Methods for Long-Time Numerical Integration of Planetary
}

\section{Orbits}

\author{
HIROSHI KINOSHITA AND HIROSHI NAKAI \\ National Astronomical Observatory \\ 2-21-1 Osawa,Mitaka, Tokyo 181, Japan \\ E-Mail address:Kinoshita@c1.mtk.nao.ac.jp
}

\begin{abstract}
When planetary orbits are numerically integrated for a long time by conventional integrators, the most serious problem is secular errors in the energy and the angular momentum of the planetary system due to discretization (truncation) errors. The secular errors in the energy and the angular momentum mean that the semi-major axes, the eccentricities, and the inclinations of planetary orbits have a secular error which grows linearly with time. Recently symplectic integrators and linear symmetric multistep integrators are found not to produce the secular errors in the energy and the angular momentum due to the discretization errors. Here we describe briefly both methods and discuss favorable properties of these integrators for a long-term integration of planetary orbits.
\end{abstract}

Key words: Numerical Integration - Symplectic Integrators - Linear Symmetric Multistep Integrators - Planetary Orbits

\section{Introduction}

With a rapid increase of computer technology, the time span of a planetary orbit simulation is increasing from one million years( Cohen,Hubbard, and Oesterwinter 1973 ) to one billion years( Sussman and Wisdom 1988). Now with use of a recent hardware of computer, it is not impossible to integrate the planetary system for the age of the solar system. As the integration time increases, the numerical results are more polluted due to various errors, which Millani and Nobili (1988) discussed in detail. Some of them are discretization (truncation) errors that are caused by the replacement of continuous differential equations by finite difference equations, round-off errors that are originated in the limit of the word length of a digital computer, and errors in the physical model used in the differential equations for the planetary system. Among them the most serious error is the accumulation of the discretization error due to a finite stepsize. The conventional integrators such as Runge-Kutta methods, multistep methods, Taylor methods, cause linear secular errors to the energy and the angular momentum which means that the semi-major axis, the eccentricity, and the inclination change linearly with time and the linear secular error in the semi-major axis produces a quadratically secular error in the planetary longitude. Therefore it is quite difficult to discuss the stability of the planetary system from a long term integration which is suffered from the spurious errors mentioned above.

On the other hand symplectic integrators (Ruth 1983,Neri 1988, or see more detail in Kinoshita et al. 1991) do not produce secular truncation errors in the actions of a Hamiltonian system, which means that the total energy of the Hamiltonian do not have a secular truncation error and the truncation error in longitude grows only linearly with time. This remarkable feature of the symplectic integrators is mathematically proved by Yoshida (1990a). Recently Quinlan and Tremaine (1990) found linear symmetric multistep integrators produce only a linear secular 
truncation error in the planetary longitude, but they did not give a mathematical proof for this fact. Here we give a mathematical proof that symmetric multistep integrators do not produce a quadratic secular error in longitude in Section 3.

So far a computer time has been one of obstacles to carry out a long integration. Now this situation is drastically changed by an appearance of a high-speed and less expensive work station and a special purpose computer(ex. Digital Orrery(Sussuman and Wisdom 1988) and GRAPE(Sugimoto et al. 1990)), and the computer time is now a minor problem. A very expensive super computer is not effective for a few body problem like the planetary system, since the vectorization of a few body problem is quite low compared with a many-body problem like a globular cluster system.

\section{Explicit Symplectic Integrators}

We assume that a Hamiltonian with an arbitrary number of degrees of freedom has the following form :

$$
F(p, q)=K(p)+U(q)
$$

where $q$ and $p$ are canonical sets. In the following algorithm, the separation of $p$ and $q$ in the Hamiltonian is essential and a conservative Newtonian dynamical system is described in the form of the Hamiltonian (1). However a disturbed Hamiltonian with the following form can be treated with the same algorithm (Kinoshita et al. 1991):

$$
F(p, q)=F_{0}(p, q)+R(q)
$$

where $F_{0}(p, q)$ is an unperturbed and solvable part and $R(q)$ is a perturbed potential. Symplectic Runge-Kutta integrators for a more general form of $F(p, q)$ are discussed by Sanz-Serna(1991).

A general $k$-stage symplectic integrator is expressed as

$$
q_{i}=q_{i-1}+c_{i} h \frac{\partial K}{\partial p}\left(p_{i-1}\right), p_{i}=p_{i-1}-d_{i} h \frac{\partial U}{\partial q}\left(q_{i}\right), i=1, \ldots, k .
$$

Here $h$ is a step-size and $q_{0}$ and $p_{0}$ are initial values, and $q_{k}$ and $p_{k}$ are the numerical solution after $h$.

As for a four stage method of fourth order, the numerical coefficients $c_{i}$ and $d_{i}$ in the formula are determined from the requirement that the local truncation error is of order $h^{5}$. However they are not uniquely determined. If we require the time reversibility of the numerical solution, we can determine them uniquely:

$$
c_{1}=c_{4}=\frac{1}{2(2-\beta)}, c_{2}=c_{3}=\frac{(1-\beta)}{2(2-\beta)}, d_{1}=d_{3}=\frac{1}{(2-\beta)}, d_{2}=\frac{-\beta}{(2-\beta)}, d_{4}=0,
$$

where, $\beta=2^{1 / 3}$ (Forest 1987, Neri 1988, and Kinoshita et al. 1991).

There does not exist a $k$-stage method of order $k(k>4)$ like Runge-Kutta methods. The highest attainable order of a $k$-stage method is not known. Recently Yoshida(1990b) has found a practical method to determine the coefficients of higher 
order explicit symplectic integrators. The coefficients $c_{i}$ and $d_{i}$ for the integrators higher than fourth order are not unique even if we require the time reversibility of the integrators. Yoshida (1990b) actually found three sets for 6-th order and five sets for 8-th order by numerically solving nonlinear sets of algebraic equations. These 6-th order symplectic integrators require 7 evaluations of the force functions, the same number as the explicit 6-th order Runge-Kutta integrators.

\subsection{NumERICAL EXAMPLES}

In order to see the properties of the symplectic integrators we at first choose the two-body problem whose analytical solution is known. Figure 1 shows errors in Keplerian elements over 2000 revolutions, where initial conditions are the semimajor axis $=1$, the eccentricity $=0.1$, the inclination $=20^{\circ}$, the argument of pericenter $=20^{\circ}$, the longitude of node $=20^{\circ}$, the mean anomaly $=20^{\circ}$, and the step size $h=0.01, \mu=1$. The solid lines are by the 6 -th order symplectic integrator (SI6A in Kinoshita et al. 1991) and the dotted lines by the 6-th order Runge-Kutta integrator (Butcher 1964). The errors in the semi-major axis and the eccentricity by the Runge-Kutta integrator grow in proportion to the first order of time, and the error in the mean anomaly increases in proportion to the square of time. The discretization errors in the semi-major axis and the eccentricity by the symplectic integrator show only periodic changes. The truncation error in the mean anomaly and the argument of pericenter grows linearly in time. The secular discretization error in the mean anomaly is also caused from the fact that the mean value of the discretization error in the energy is not zero, and this mean value depends on the initial values and especially on the mean anomaly at the epoch and the accumulation of truncation errors by symplectic integrators can be treated by a canonical perturbation method (Kinoshita et al. 1991).

The errors in the inclination and the longitude of node by the symplectic integrator are not discretization errors but round-off errors, since the symplectic integrator does conserve the angular momentum vector exactly. Even if the Runge-Kutta integrator does not conserve the angular momentum vector, it conserves the orientation of the angular momentum vector, as seen in Figure 1.

The secular errors in the pericenter by both 6 -th order methods have a same order as seen in Figure 1. As for 4-th order methods, however, the secular errors in the pericenter by a 4-th order Runge-Kutta method are much smaller than those in a 4-th order symplectic integrator (Kinoshita et al. 1991), whose mathematical or dynamical explanation is not known.

We integrated five outer planets with 50 days step size for 500000 years by the symplectic integrator SI6A and the 13-th order Störmer's integrator, which were used in the numerical integration of the five outer planets by Cohen et al. (1978), Kinoshita and Nakai (1984), Applegate et al. (1986), and Sussman and Wisdom (1988). In order to reduce the round-off errors, we performed these integrations with a quadruple precision arithmetic. The errors in the total energy and the total angular momentum, which are integrals in this dynamical system, are shown in Figure 2. The results by the symplectic integrator does not show any secular trend, but Störmer's integrators clearly show a secular trend that is proportional to time. 

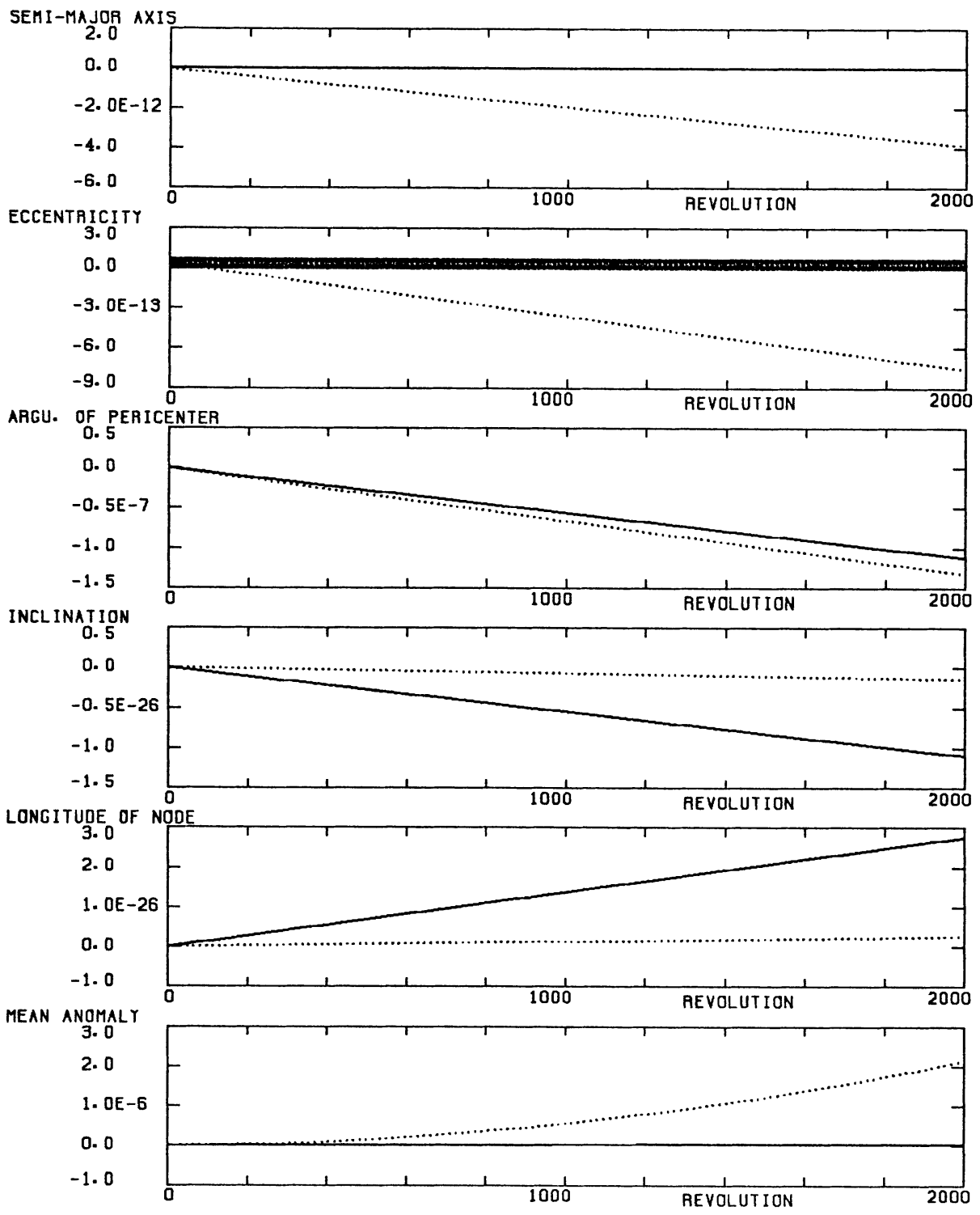

Fig. 1. Numerical errors in Keplerian elements over 2000 revolutions integration of the two-body problem by 6-th order symplectic integrator ( solid lines ) and 6-th order Runge-Kutta integrator (dotted lines). The units for angular variables are degree. 


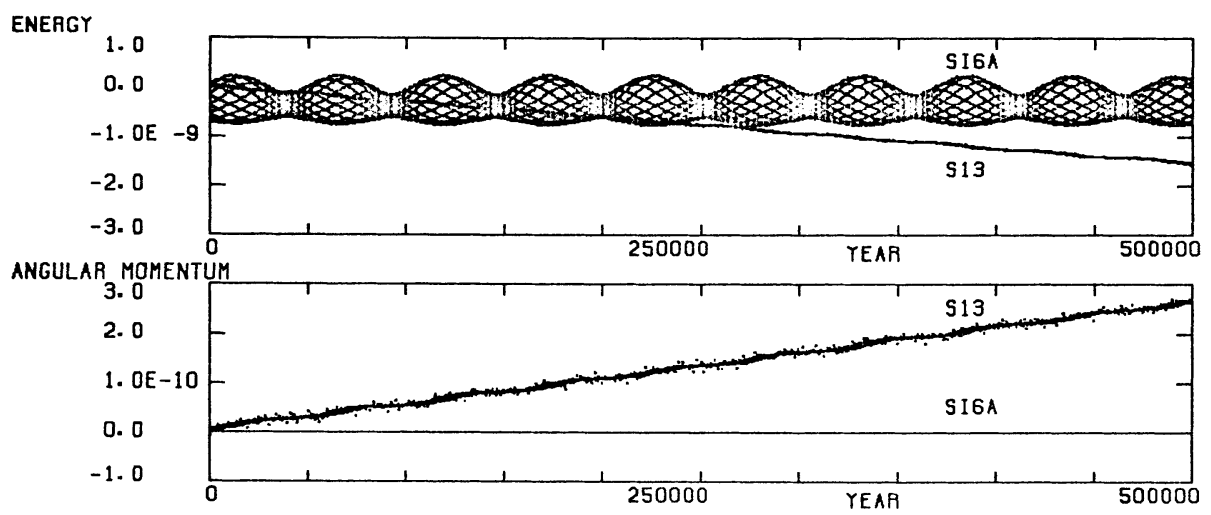

Fig. 2. Numerical errors in the total energy and the total angular momentum $\left(\triangle E / E_{0}\right.$ and $\left.\triangle A / A_{0}\right)$ in the numerical integrations of outer five planets with 6-th order symplectic integrator ( SI6A) and 13-th order Störmer's integrator(S13).

However the computer time with SI6A is about 5 times longer than that with Störmer's integrators, since the number of evaluations of the force function in SI6A is 7 and on the other hand that of Störmer's method is only one per one step. The symplectic integrators have a capability to integrate planetary systems in the time scale of $5 \times 10^{9}$ years without being polluted by the truncation errors, if we can reduce the round-off errors with use of quadruple precision arithmetics or a newly proposed method by Quinn and Tremaine(1990).

\section{Linear Symmetric Multistep Integrators}

We consider a system of the second-order differential equations

$$
\frac{d^{2} \mathbf{x}}{d t^{2}}=\mathbf{f}(\mathbf{x}, t)
$$

where $\mathbf{x}=\left(x_{1}, \ldots, x_{n}\right), \mathbf{f}=\left(f_{1}, \ldots, f_{n}\right)$. The numerical solutions by $k$-th step multistep integrators are computed recursively by the following difference equation:

$$
\alpha_{k} \mathbf{X}_{n+k}+\ldots+\alpha_{0} \mathbf{X}_{n}=h^{2}\left(\beta_{k} \mathbf{f}\left(\mathbf{X}_{n+k}, t+k h\right)+\ldots+\beta_{0} \mathbf{f}\left(\mathbf{X}_{n}, t\right)\right)
$$

where the coefficients $\alpha$ and $\beta$ are independent of the form $f$. The local truncation error associated with the difference equation (5) is

$$
L[\mathbf{x}(t) ; h]=C_{p+2} h^{p+2} \mathbf{x}^{(p+2)}(t)+C_{p+3} h^{p+3} \mathbf{x}^{(p+3)}(t)+O\left(h^{p+4}\right),
$$

where $p$ is the order of the difference equation(5), and $C_{p+2}, C_{p+3}$ are functions of $\alpha$ and $\beta$.

Symmetric multistep integrators (Henrici 1962) are defined as

$$
\alpha_{s} \mathbf{X}_{n+s}+\alpha_{s-1} \mathbf{X}_{n+s-1}+\ldots+\alpha_{-s} \mathbf{X}_{n-s}=
$$




$$
h^{2}\left(\beta_{s} \mathbf{f}\left(\mathbf{X}_{n+s}, t+s h\right)+\ldots+\beta_{-s} \mathbf{f}\left(\mathbf{X}_{n-s}, t-s h\right)\right),
$$

where $\alpha_{\mu}=\alpha_{-\mu}, \beta_{\mu}=\beta_{-\mu}, \mu=0, \ldots, s, k=2 s$. Henrici(1962) showed that the order $p$ of the difference equation(5) is even. The local truncation error for (7) is expressed as

$$
L[\mathbf{x} ; h]=C_{p+2} h^{p+2} \mathbf{x}^{(p+2)}(t)+C_{p+4} h^{p+4} \mathbf{x}^{(p+4)}(t)+O\left(h^{p+6}\right),
$$

which is easily derived with use of the symmetry of $\alpha$ and $\beta$. It should be noted that $L[\mathbf{x}(t) ; h]$ does not have odd order derivatives of $\mathbf{x}(t)$ with respect time, which is the most important point in the discussion of the secular truncation errors. This symmetry also gives an exact time reversibility to a symmetric multistep integrator if a round-off error is neglected. Quinlan and Tremaine (1990) found symmetric integrators produce only a linear secular truncation error in the planetary longitude, and they did not give a mathematical explanation for this fact. In the following we discuss the global truncation errors of symmetric multistep integrators.

The truncation error $\delta \mathbf{x}=\mathbf{X}-\mathbf{x}$, where $\mathbf{X}$ is the solution of the difference equation (5) and $\mathbf{x}$ is the solution of the original differential equations (4), is given by Henrici(1962):

$$
\delta \mathbf{x}(t)=\mathbf{e}(t) h^{p}+\mathbf{d}(t)
$$

where $\mathbf{e}(t)$ is defined by

$$
\frac{d^{2} \mathbf{e}}{d t^{2}}=G(t) \mathbf{e}-E_{1} \mathbf{x}^{(p+2)}(t)+O(h), \mathbf{e}(0)=\mathbf{e}^{\prime}(0)=0
$$

with $G(t)=\partial \mathbf{f}(\mathbf{x}, t) / \partial \mathbf{x}$. The coefficient $E_{1}$ of the second term in (10) is related to the first term of $(6)$ and defined by

$$
E_{1}=C_{p+2} /\left(\beta_{k}+\ldots+\beta_{0}\right) .
$$

The third term in (10) comes from the second and third terms of (6). The first term of the truncation error (9) is the genuine term that is inevitable in the numerical computation, and the second term $\mathrm{d}(t)$, which satisfies a similar equation to (10)(Henrici 1962), is due to errors in starting values. If the starting values are determined precisely, the second term can be neglected. We, therefore, neglect the second term in the following discussions. Even if the starting values have errors, the second term does not produce a secular error in the Keplerian elements of the two-body problem (Kinoshita 1968).

Now we discuss the accumulation of truncation errors for the two-body problem with use of the equation (10). The equation (10) is linear but non-homogeneous. The homogeneous part of (10) is the variational equation of the two-body problem . Since the general solutions of the two-body problem are expressed analytically, the general solutions of the variational equations are easily obtained by differentiating the general solutions with respect to the six Keplerian elements and the solution of the non-homogeneous linear equation (10) is analytically solved ( see more detail in Kinoshita 1968). 
With use of results and similar methods by Kinoshita(1968), who discussed the truncation error for the first-order differential equation, the truncation error in the true longitude $\lambda$ is expressed as

$$
\begin{aligned}
\delta \lambda /\left(-E_{1} h^{p}\right)= & -\frac{3}{2} \frac{a \sqrt{1-e^{2}}}{r^{2}} l A^{p}+\frac{\sin f(2+e \cos f)}{1-e^{2}} B^{p} \\
& -\frac{a^{2} n \sqrt{1-e^{2}}}{r^{2}} C^{p}-2 \sqrt{1-e^{2}}(2+e \cos f) \cos f D^{p},
\end{aligned}
$$

where,

$$
\begin{aligned}
& A^{p}=2 \int_{t_{0}}^{t}\left(-\frac{\sin f}{n \sqrt{1-e^{2}}} x^{*(p+2)}(t)+\frac{\cos f+e}{n \sqrt{1-e^{2}}} y^{*(p+2)}\right) d t \\
& B^{p}=\int_{t_{0}}^{t}\left(-\frac{r \sin f(\cos f+e)}{a^{2} n \sqrt{1-e^{2}}} x^{*(p+2)}(t)+\right. \\
& \left.\frac{r\left(1+\cos ^{2} f+2 e \cos f\right)}{a^{2} n \sqrt{1-e^{2}}} y^{*(p+2)}(t)\right) d t, \\
& C^{p}=C_{1}^{p}+C_{2}^{p} \text {, } \\
& C_{1}^{p}=3 \int_{t_{0}}^{t}\left(\frac{\sin f}{a n^{2} \sqrt{1-e^{2}}} l x^{*(p+2)}(t)-\frac{\cos f+e}{a n^{2} \sqrt{1-e^{2}}} l y^{*(p+2)}(t)\right) d t, \\
& C_{2}^{p}=\int_{t_{0}}^{t}\left(\frac{r(\cos f-e)(2+e \cos f)}{a^{2} n^{2}} x^{*(p+2)}(t)+\right. \\
& \left.\frac{r \sin f(2+e \cos f)}{a^{2} n^{2}} y^{*(p+2)}(t)\right) d t, \\
& D^{p}=\int_{t_{0}}^{t}\left(-\frac{r(3-\cos 2 f+2 e \cos f)}{4 a n^{2}\left(1-e^{2}\right)} x^{*(p+2)}(t)+\right. \\
& \left.\frac{r \sin 2 f}{4 a n^{2}\left(1-e^{2}\right)} y^{*(p+2)}(t)\right) d t
\end{aligned}
$$

with

$$
x^{*}=a(\cos u-e), y^{*}=a \sqrt{1-e^{2}} \sin u .
$$

Here $a, e$, and $n$ are the semi-major axis, the eccentricity, and the mean motion, and $f, u$, and $l$ are the true, eccentric, and mean anomaly. The function $x^{*}$ is an even periodic function with respect to time and $y^{*}$ is an odd periodic function. At first we discuss the case where the order $p$ is even. The function $x^{*(p+2)}$ is an even periodic function and $y^{*(p+2)}$ is an odd periodic function. The coefficients of $x^{*(p+2)}$ and $y^{*(p+2)}$ in the inside of the integral (13) are odd and even,respectively. Therefore the inside of the integral (13) is an odd periodic function, and, therefore, 


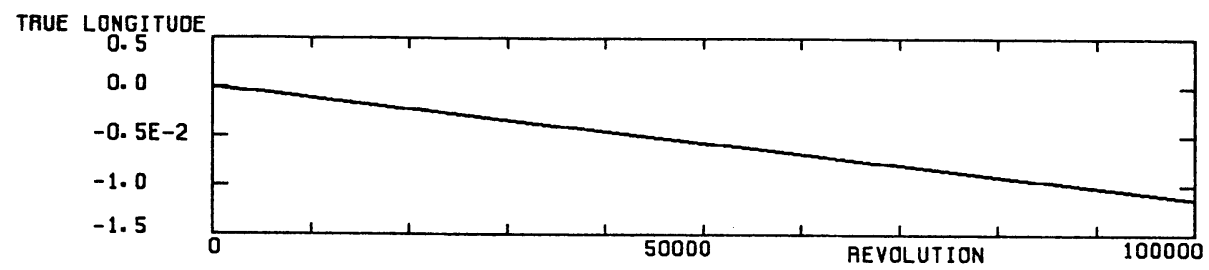

Fig. 3. Truncation errors of the true longitude of the two-body problem for 100,000 revolutions by 12 th-order symmetric multistep integrator. The unit is arcsecond.

$A^{(p)}$ is an even periodic function without a secular term. Similarly $B^{(p)}$ is an even periodic function without a secular term, and both $C^{(p)}$ and $D^{(p)}$ are odd periodic functions with a linear secular term. When the order $p$ is odd, $A^{(p)}$ and $B^{(p)}$ have a linear secular term, $C^{(p)}$ has a quadratic secular term, and $D^{(p)}$ does not have a secular term.

The order $p$ for the symmetric multistep integrators is even as described before, and, therefore, the linear secular truncation error in the longitude does appear from the first,third, and fourth terms in (12), but the quadratic secular errors does not appear. The truncation error from the third term of (10), whose order is $O\left(h^{2}\right)$ for the symmetric integrators, does not produce the quadratic secular errors in the longitude, since the second and thirds terms of (8) include only even order derivatives of $\mathbf{x}(t)$ with respect to time. The linear secular error from these terms is smaller by order $h^{2}$ than the principal linear secular error.

We applied the 12th-order symmetric integrator constructed by Quinlan and Tremaine (1990) to the two-body problem and integrated an orbit $(a=1.0, e=$ $0.05, l=0.0, \omega=0.0$ ) for 100,000 revolutions with a stepsize $h=0.009228 \times$ $2 \pi$. The orbital elements and the stepsize in this example were used by Quinlan and Tremaine(1990), and the eccentricity is close to Jupiter's one and the stepsize corresponds to a 40 day stepsize for Jupiter. In order to reduce round-off errors we carried out the numerical integration with a quadruple precision arithmetic. Figure 3 show the residuals of the true longitude that grows linearly with time.

The variations of Keplerian elements of this orbit are shown in Figure 4. The semi-major axis and the eccentricity do not have a secular trend at all.

Then we integrated the five outer planets for 10,000 years by the same symmetric integrator and 13th-order Störmer multistep integrator. Also we integrated the outer five planets by the extrapolation method developed by Gragg (1965) with a quadruple precision arithmetic as a standard orbit for comparison. The accuracy of the standard orbit is checked by the time reversibility of the numerical solution: we integrated the motion over 10,000 years in backward with use of the positions and the velocity at the final epoch of the forward integration and examined how the positions at the initial epoch are close to the initial positions. The differences in the longitudes between forward and backward integrations increase quadratically with time and the maximum difference in the longitude of Jupiter is only $3 \times 10^{-8}$ arcsecond. The standard orbit thus obtained is accurate enough to test the precision 

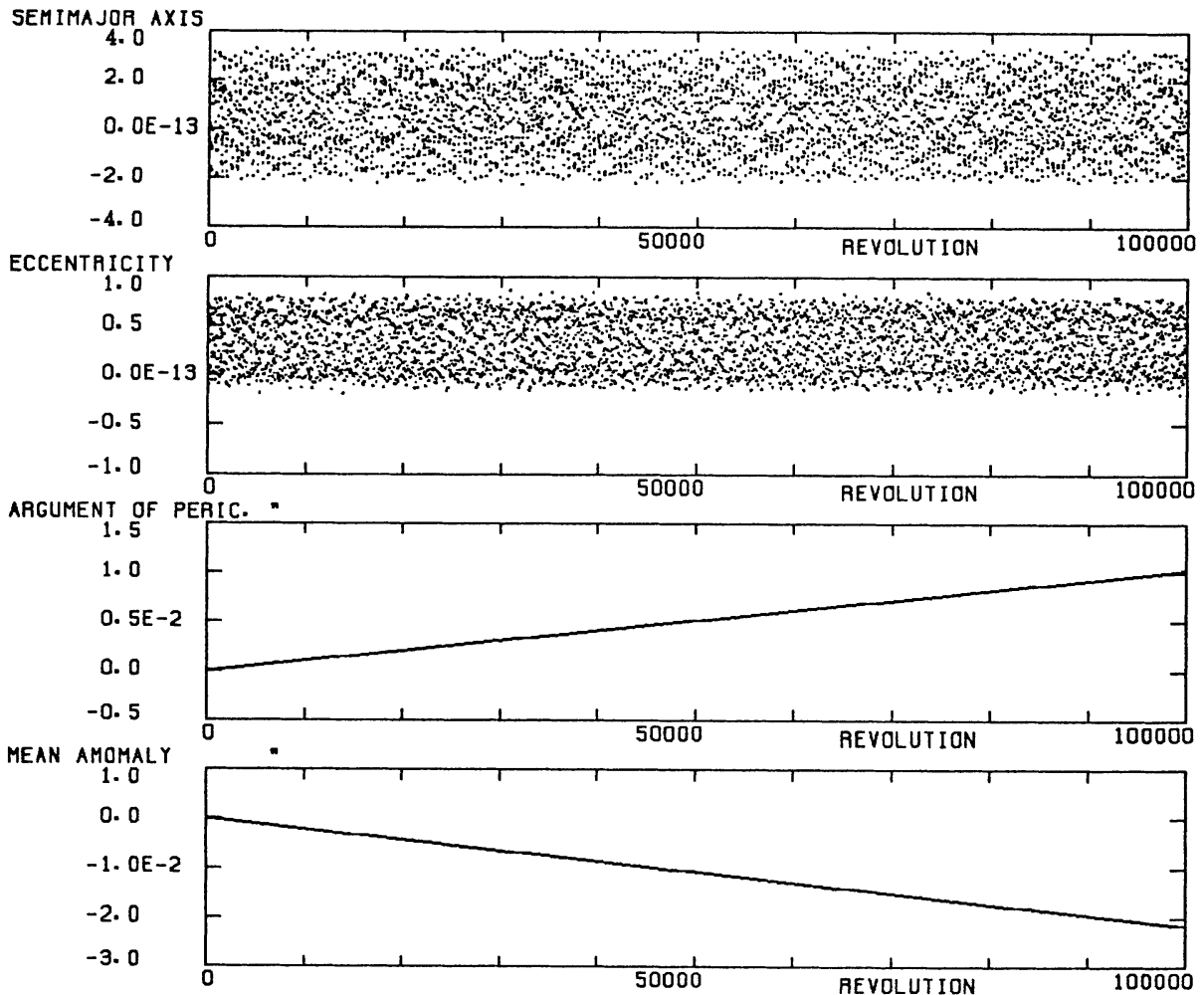

Fig. 4. Truncation errors of the Keplerian elements of the two-body problem for 100,000 revolutions by 12 th-order symmetric multistep integrator. The units for angular variables are arcsecond.

A)

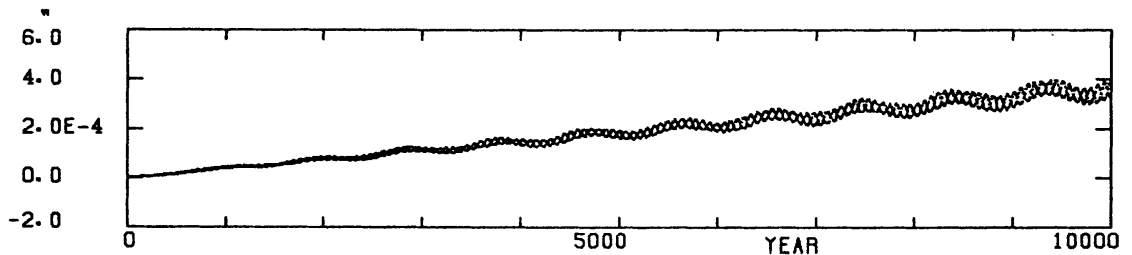

B]

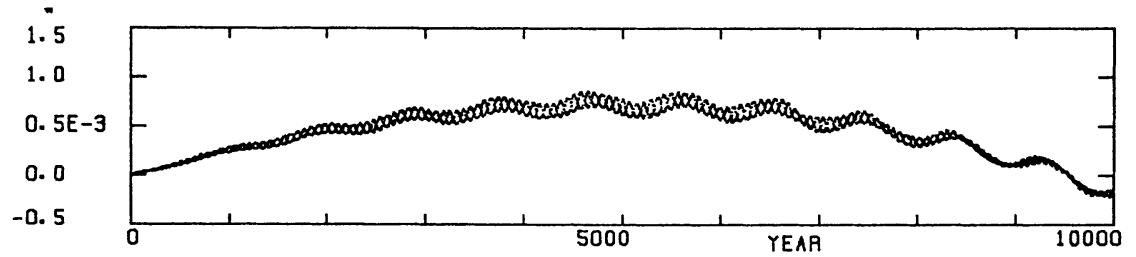

Fig. 5. Truncation errors of Jupiter's longitude in the numerical integration of outer five planets by two multistep integrators: (the unit is arcsecond)

A)12th-order symmetric multistep integrator,

B)13th-order Störmer multistep integrator. 
of the orbits integrated by the multistep methods. Figure 5 shows the residuals in Jupiter's longitude. The truncation error of the symmetric method increases linearly with time, and that of the Störmer integrator grows quadratically with time.

Here we give a short comment on one-leg method which is one version of linear multistep methods and becomes symplectic. We introduce two polynomials with coefficients $\alpha$ and $\beta$ :

$$
\rho(E)=\sum_{j=0}^{k} \alpha_{j} E^{j}, \sigma(E)=\sum_{j=0}^{j} \beta_{j} E^{j},
$$

where $E$ denotes the shift operator $E y_{n}=y_{n+1}$. Let us consider a first-order differential equation

$$
\frac{d x}{d t}=f(x) \text {. }
$$

Linear multistep method for (21) is express as

$$
\rho(E) x_{n}=h \sigma(E) f\left(x_{n}\right) .
$$

Dahlquist(1976) introduced a new type of integrators with use of the same $\rho$ and $\sigma$ :

$$
\rho(E) x_{n}=h f\left(\sigma(E) x_{n}\right)
$$

and called it the one-leg method associated with (22). Eirola and Sanz-Serna(1990) showed that if the linear multistep method is symmetric, the corresponding one-leg method is canonical when applied to Hamiltonian systems of differential equations.

\section{Summary}

The merits of symplectic integrators are summarized as follows:

1. the numerical solutions have a property of area preserving,

2. the energy integral does not have a secular truncation error, which means that the longitude error grows linearly with the time instead of quadratic growth,

3 . the angular momentum vector of n-body problem is exactly conserved,

4. the numerical solution has a property of time reversibility, when the coefficients of integrators are symmetric,

5. the global truncation errors,especially the secular error in the angle variables, can be easily estimated by an canonical perturbation method.

The demerit of the symplectic integrators for a long integration is time-consuming, since the number of evaluations of the force function in the symplectic integrators is larger than the traditional integrators. For example, the symplectic integrators with order 6 require seven evaluations of the force function per one step and on the other hand multistep integrators of any order require only one evaluation of the force function per one step.

The merits of linear symmetric multistep integrators are

1. the longitude error grows linearly with the time instead of quadratic growth,

2. the energy and the angular momentum do not have a secular truncation error, 
3. the numerical solution has a property of time reversibility,

4. the number of evaluations of the force function is only one per one step, which means the computer time with linear multistep integrators is shorter than with symplectic integrators.

Recently Quinlan and Toomre (1991) found a dangerous character of symmetric integrators: at special stepsizes they exhibit instabilities that result from resonances between the orbital motion and one or more spurious roots of the method itself. However, Quinlan (1991) thinks that since the resonances are rare and are easy to avoid, the symmetric method is still useful provided that it is used with some caution.

\section{References}

Applegate, J.H., Douglas, M.R., Gürsel, Y., Sussman, G.J. and Wisdom, J.: 1986, 'The Outer Solar System for 200 Million Years', A stron. J. 92, 176-194.

Butcher,J.C.: 1964, 'On Runge-Kutta processes of higher order', J.A ustral.Math.Soc. 4, $179-194$.

Cohen, C.J., Hubbard, E.C., and Oesterwinter, C.: 1973, 'Elements of the Outer Planets for One Million Years', Astron. Papers Amer. Ephemeris 92, 3-92.

Dahlquist,G.: 1976, 'On One-Leg Multistep Method', SIAM J.Numer.Anal. 20, 1130-1138.

Eirola,T. and Sanz-Serna,J.M.: 1990, 'Conservation of Integrals and Symplectic Structure in the Integration of Differential Equations by Multistep Methods', Applied Mathematics and Computation Reports, Universidad de Valladolid Report 1990/9, pp.9.

Gragg,W.: 1963, 'Repeated Extrapolation to the Limit in the Numerical Solution of Ordinary Differential Equations', Doctoral Dissertation,University of California,Los Angels, pp.103.

Henrici,P.: 1962, Discrete Variable Methods in Ordinary Differential Equations, Wiley, New York.407.

Kinoshita,H.: 1968, ' Propagation of the Discretization Error in the Two-Body Problem ', Publ.Astron.Soc.Japan 20, 1-23.

Kinoshita, H. and Nakai, H.: 1984, 'Motions of the Perihelions of Neptune and Pluto', Celest. Mech. 34, 203-217.

Kinoshita, H.: 1989, 'Numerical Integration Method in Dynamical Astronomy', Celest. Mech. 45, 231-244.

Kinoshita,H.,Yoshida,H.,and Nakai,H.: 1991, 'Symplectic Integrators and Applications to Dynamical Astronomy', Celest.Mech. 50, 59-71.

Kinoshita,H. and Nakai,H.:1991, 'Error Analysis of Symmetric Integrators for Planetary Orbits', in The Twenty-Fourth Symposium on Celestial Mechanics, ed. by H.Kinoshita and H.Yoshida,139-147.

Milani,A. and Nobili,A.: 1988, 'Integration Error over Very Long Time Spans', Celest.Mech. 43, 1-34.

Neri, F.: 1988, 'Lie Algebras and Canonical Integration', University of Maryland, Dept.of Physics , preprint.

Quinlan,D and Tremaine,S.: 1990, 'Symmetric Multistep Methods for the Numerical Integration of Planetary Orbits', Astron.J. 100, 1964-1700.

Quinlan, G.D. and Toomre, A.T. : 1991, 'Resonant Instabilities in Symmetric Multistep Methods' , private communication.

Quinlan,D.:1991,private communication.

Quinn, T. and Tremaine, S.: 1990, 'Roundoff Error in Long-Term Planetary Orbit Integrals', Astron.J. 99, 1016-1023.

Ruth, R.: 1983, 'A Canonical Integration Technique', IEEE Trans. Nucl. Sci. 30, (4), $2699-2671$.

Sanz-Serna,J.M.: 1991, 'Symplectic Runge-Kutta and Related Methods:Recent Results', Applied Mathematics and Computation Reports, Universidad de Valladolid Report 1991/5, pp.11.

Sugimoto,D., Chikada,Y., Makino,J., Ito,T., Ebisuzaki,T., and Umemura,M.: 1990, 'A SpecialPurpose Computer for Gravitational Many-Body Problems', Nature 345, 33-35.

Sussman, G.J. and Wisdom, J.: 1988, 'Numerical Evidence That the Motion of Pluto is Chaotic', Science 241, 433-437. 
Yoshida, H.: 1990a, 'Conserved Quantities of Symplectic Integrators for Hamiltonian Systems', Physica $D$, in press.

Yoshida,H.: 1990b, 'Construction of Higher Order Symplectic Integrators', Phys.Lett.A. 150, 262268.

\section{Discussion}

J.Laskar - Do I understand that you want to abandon symplectic integrator for long term integrations of the solar system?

H.Kinoshita - If symmetric multistep methods do not have instabilities discovered by Quinlan, I'll use symmetric integrators rather than symplectic integrators from the economical point of view of CPU time. If one-leg methods have the same instabilities as the corresponding symmetric integrators, then I'll use symplectic integrators whose mathematical characters are well understood. 